\title{
Tongue Strength Training Increases Daytime Upper Airway Stability in Rats
}

\section{Hong Huang \\ Wenyang $\mathrm{Li}$ \\ Hongyu Jin \\ Lei Zhang \\ Zhijing Wei \\ Wei Wang}

Institute of Respiratory and Critical Care, The First Hospital of China Medical University, Shenyang, People's Republic of China
Correspondence: Wei Wang Institute of Respiratory and Critical Care, The First Hospital of China Medical University, 155 Nanjing North Street, Heping District, Shenyang, Liaoning, I I000I, People's Republic of China Tel +862483282529

Fax +862483282002

Email wwbycmu@I26.com
Purpose: Tongue strength training (TST) has been shown to decrease the apnea-hypopnea index in some patients with obstructive sleep apnea (OSA). However, whether TST modulates the central regulation of genioglossus and influences the stability of the upper airway remains unknown. The purpose of this study was to dynamically assess the effect of TST on the upper airway.

Methods: Sixteen adult male Sprague-Dawley rats were studied to explore the mechanism of TST improving the upper airway function. The rats were randomly assigned to the normal control (NC) and TST groups. The TST group underwent 8-week progressive resistance tongue exercise training. Transcranial magnetic stimulation (TMS) responses and EMG activities were consistently recorded for $2 \mathrm{~h}$ on days $0,14,28$, and 56 of the experiments in both groups. Theoretical critical pressure (Pcrit) value was measured on days $0,14,28$, and 56.

Results: The TST group showed shorter TMS latency and higher genioglossus EMG activity, which lasted from $5 \mathrm{~min}$ to $80 \mathrm{~min}$ after training on day 56 of training, than the NC group. The TST group showed significantly lower theoretical Pcrit values on days 28 and 56 of training than the $\mathrm{NC}$ group $\left(-4.07 \pm 0.92\right.$ vs $-3.12 \pm 0.77 \mathrm{cmH}_{2} \mathrm{O}, P<0.05,-4.66 \pm 0.74$ vs $\left.-3.07 \pm 0.38 \mathrm{cmH}_{2} \mathrm{O}, P<0.01\right)$.

Conclusion: This study revealed that an 8-week TST could gradually and transiently increase corticomotor excitability of genioglossus, elevate the genioglossus EMG activity, and ultimately enhance the stability of the upper airway during daytime. Moreover, improved neuromuscular excitability occurred prior to the enhanced upper airway stability. These findings provide a theoretical foundation for TST as a promising treatment for OSA patients. Keywords: tongue strength training, genioglossus, upper airway critical pressure, transcranial magnetic stimulation, corticomotor excitability

\section{Introduction}

Obstructive sleep apnea (OSA) characterized by repetitive upper airway collapse only during sleep. As one of the typical upper airway dilator muscles, genioglossus plays a key role in maintaining upper airway patency. It is well known that OSA patients show decreased genioglossus EMG activity when compared to normal subjects during sleep onset. ${ }^{1}$ Eckert et $\mathrm{al}^{2}$ further reported that over one-third of OSA patients showed a less than $0.1 \%$ of maximum increase in EMG activity per centimeter of water of negative epiglottic pressure during sleep. Thus, dysfunction of the upper airway dilator muscles is particularly important in the pathogenesis of OSA. Interestingly, OSA patients showed higher waking EMG activities of genioglossus than healthy control subjects. ${ }^{3}$ Our previous studies also observed 
increased responsiveness of genioglossus to transcranial magnetic stimulation (TMS) in patients with OSA compared to that in normal subjects during wakefulness. ${ }^{4,5}$ However, awake upper airway collapsibility was still remarkably higher in patients with OSA than in normal subjects. ${ }^{6}$ These findings indicated that daytime neuromuscular compensation was present in OSA patients, but it was incomplete.

Therefore, as oropharyngeal training focused on improving the contractility of upper airway dilator muscles, could it be a promising therapy for OSA patients? The results from a meta-analysis showed that oropharyngeal exercises decrease the apnea-hypopnea index (AHI) in mild-to-moderate OSA patients when compared to sham therapy, but the certainty of the evidence is low. ${ }^{7}$ Verma et $\mathrm{al}^{8}$ found that 3-month oropharyngeal exercise therapy could significantly improve the minimum oxygen saturation and time duration of $\mathrm{SaO} 2<90 \%$ of OSA patients, while the average AHI of OSA patients after training was similar to pre-treatment. Many studies indicated that progressive tongue strength training (TST) could elevate tongue strength and functional dietary intake by mouth in older individuals. ${ }^{9,10}$ Only a pilot study showed that AHI dropped from 20.9 to 16.1 events per hour after a 7-day tongue-task exercise in 10 mild-tomoderate OSA patients. ${ }^{11}$ Previous studies also found that muscular resistance training can increase muscle EMG activity and alter central excitability. ${ }^{12}$ However, whether training evokes central plasticity first or preferentially elevates muscle EMG activity has not been systematically explored. Critical pressure (Pcrit) is the gold standard to quantify upper airway collapsibility. Higher Pcrit was observed in OSA patients than in non-OSA subjects, and the increased Pcrit was positively correlated with the severity of OSA. ${ }^{13}$ Our previous study also revealed that chronic intermittent hypoxia increased the theoretical Pcrit value of the upper airway in rats, although it simultaneously induced central compensation of genioglossus. ${ }^{14}$ However, to date, few experiments have been performed to observe the changes in central compensation and peripheral stability of the upper airway during oropharyngeal training. In order to explore the mechanism of TST improving the upper airway function, we assessed the impact of TST on the genioglossus corticomotor excitability, genioglossus EMG activity, and upper airway collapsibility of rats at different phases of training, and further analyzed the interaction among them.

\section{Materials and Methods}

\section{Animals}

Adult male Sprague-Dawley rats (240-280 g) were obtained from the Liao Ning Chang Sheng Biotechnology Company (Benxi, China). The rats were randomly assigned to the normal control (NC) group $(\mathrm{n}=8)$ and the TST group $(\mathrm{n}=8)$. Rats were individually caged under controlled temperature, relative air humidity, and $12 \mathrm{~h}$ light-dark cycle. Water was limited to $3 \mathrm{~h}$ per day, while food was available ad libitum during the training intervention. All procedures were carried out after approval by the animal's Ethics and Use Committee of China Medical University and in compliance with the NIH Guide for Care and Use of Animals.

\section{Tongue Strength Training}

TST was performed using a custom instrument (Figure 1), which was designed based on previous studies. ${ }^{15,16}$ Before the training program, all rats underwent a 7-day water restriction protocol, where the time of water availability gradually dropped from the whole day to only $3 \mathrm{~h}$ per day. The use of the instrument involved a traditional learning paradigm in which thirsty rats were trained to lick the $18 \mathrm{~mm}$ operandum disk linked with a force transducer (HBM, 0-300 g range) to obtain a water reward. In preparation for maximum voluntary tongue force (MVTF) testing and TST, a 3-day adaptation period was set for acclimation to the instrument. At the beginning of this period, water was manually distributed when rats approached the operandum disk. During the later period, only if the rats licked the disk with more than 0.2-gram strength would they get the water reward. The training groups then began 8 -week tongue training for 5 days per week. The NC group only received the water restriction protocol except for MVTF testing fortnightly. The MVTF was defined as the average of the largest ten force values obtained and recorded in two minutes when the force requirement was $2 \mathrm{~g}$. Muscle total work, defined as the total area under the curve of all the strength during daily training, was calculated using specific software and read from the computer screen to quantify the training effect. The force threshold for water access, training duration, and muscle total work increased progressively during the entire training period. Specifically, the daily training time gradually increased from $10 \mathrm{~min}$ to $30 \mathrm{~min}$. Over the first six weeks, the training time increased by five min every two weeks. The exercise durations were 25 and $30 \mathrm{~min}$ in the 


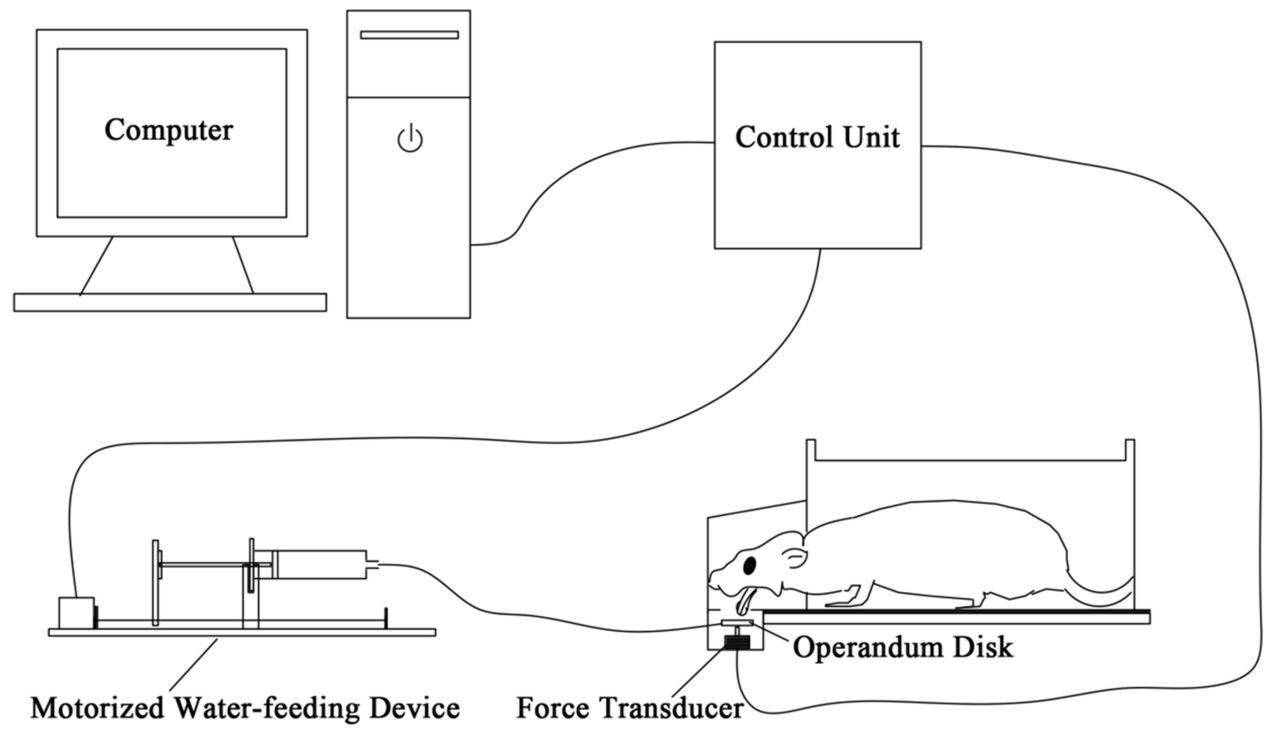

Figure I Schematic diagram of the tongue strength training operandum.

7th and 8th week, respectively (Supplementary Table 1). The TST group rats were given water ad libitum for $2 \mathrm{~h} 30$ min to $2 \mathrm{~h} 50 \mathrm{~min}$ after training to ensure the $3 \mathrm{~h}$ total water access time per day. The MVTF test was performed during the first 2 min of every training day. During the remaining time, the force threshold was $50 \%, 60 \%, 70 \%$, and $80 \%$ of MVTF measured on the corresponding day in weeks 1-2, 3-4, 5-6, and 7-8, respectively (Supplementary Table 1). The threshold of total muscle work was set at 1000, 1500, 2000, and 2500 g.s in weeks 1-2, 3-4, 5-6, and 7-8, respectively (Supplementary Table 1 ). The progression principle of exercise intensity and duration is performed following the recommendations of strength training for humans by the American College of Sports Medicine. ${ }^{17}$

\section{Transcranial Magnetic Stimulation}

TMS was performed on days $0,14,28$, and 56 of the study. The methodology is described in detail in our previous papers and briefly reviewed here. ${ }^{14,18}$ The rats were anesthetized by an intraperitoneal injection of sodium pentobarbital based on the performance of the toe-pitch withdrawal reflex, and the heads and limbs of the rats were restrained on wooden boards. Single-pulse TMS was achieved using a Magstim 200 stimulator (Magstim, Whitland, Dyfed, UK). According to previous research, the coil was fixed against the head of rats at the optimal stimulation site (defined as $2.0-4.0 \mathrm{~mm}$ from midline and $3.0-5.0 \mathrm{~mm}$ rostral to bregma). The response of the genioglossus corticomotor to TMS was recorded by inserting a concentric needle electrode (NM-131 T, Nihon Kohden,
Japan) into the genioglossus and assessed by latency and amplitude of motor-evoked potentials. TMS signals were amplified and recorded using a specialized computer software package (AxoScope software 9.0, Axon Instruments, Inc., USA). Latency was defined as the time up to the onset of the first biphasic wave following magnetic stimulation, and amplitude was measured from peak to peak of the wave (as shown in Supplementary Figure 1A). The TMS responses were recorded at $5 \mathrm{~min}, 20 \mathrm{~min}, 40$ min, $80 \mathrm{~min}$, and $120 \mathrm{~min}$ after TST. For each timepoint, three stimulations were applied with $30 \mathrm{~s}$ intervals, and the average value was calculated.

\section{Electromyogram (EMG)}

The rats were prepared as described in the TMS measurements. EMG was measured using a concentric needle electrode inserted into the genioglossus. The EMG signals were filtered (300-10,000 Hz), amplified, and integrated (JB-904BK, Nihon Kohden). An electric stimulator (Neuropack Manager, Nihon Kohden) was used to digitize and rectify the integrated signal. Typical genioglossus EMG activity is ${ }^{14}$ shown in Supplementary Figure 1B. The EMG was recorded at $5 \mathrm{~min}, 20 \mathrm{~min}, 40 \mathrm{~min}, 80$ min, and $120 \mathrm{~min}$ after TST.

\section{Theoretical Pcrit Value}

The rats were anesthetized with an intraperitoneal injection of sodium pentobarbital. The theoretical Pcrit value was measured as previously described. ${ }^{14}$ After the airway secretion was cleaned, a double-layer 3-cm-long PE cannula, the 
tip of which has a side hole, was inserted into the rats' oral cavity. The outside diameter of the outer and inner tubes was 3.7 and $2.1 \mathrm{~mm}$, respectively. The tip of the inner tube, which extended $3.7 \mathrm{~mm}$ beyond the outer tube, was placed at the boundary of the oropharynx and soft palate. The outer tube was opened in air. The other end of the inner tube was attached to the pressure and airflow transducer. Then, the downstream end of the transducer was further attached to a medical negative pressure suction device. In this way, the negative driving pressure $\left(P_{d}\right)$ and instantaneous airflow $\left(_{\mathrm{I}}\right)$ across the oropharynx were measured simultaneously. After sealing the mouths and noses of rats, the negative pressure of the upper airway was gradually increased by the suction device until the I plateaued or reduced. PowerLab software (AD Instruments, Colorado Springs, CO, USA) was used to amplify, digitize, and analyze $P_{d}$ and $\dot{V}_{\mathrm{I}}$. A second-degree polynomial regression model $\left(\mathrm{I}=K_{1} P_{d}+\mathrm{K}_{2} P_{d}^{2}\right)$ characterizing the relationship between $P_{d}$ and the $\dot{V}_{\text {I value }}\left(0_{-}\right.$I max range) was used to model the upper airway dynamic response of flow-limited twitches. When $\dot{V}_{\mathrm{I}}=0$ and $P_{d} \neq 0$, the solution of this equation is regarded as the theoretical value of Pcrit.

\section{Statistical Analysis}

The results are shown as the mean \pm standard deviation. Data were analyzed by a repeated measures two-way ANOVA with Bonferroni correction, and the grouping factors were time and training. All analyses were conducted using SPSS ver. 23.0 (SPSS, Chicago, IL, USA). Data were graphed using the GraphPad Prism 7 software. $\mathrm{P}<0.05$ was considered statistically significant.

\section{Results}

\section{Mechanical Property of Genioglossus}

The results of MVTF at different time points are shown in Figure 2. Baseline values of MVTF were similar between the two groups. The MVTF of the TST group gradually increased from day 14 to day 56 of training compared to that of the NC group.

\section{Genioglossus Activity Assessed by EMG Recording}

For baseline EMG activity of genioglossus, no significant difference was observed between the NC and TST groups at all time points (Figure 3A). On day 14, EMG activity of the genioglossus significantly increased from $5 \mathrm{~min}$ to 20 min after tongue training in the TST group compared to

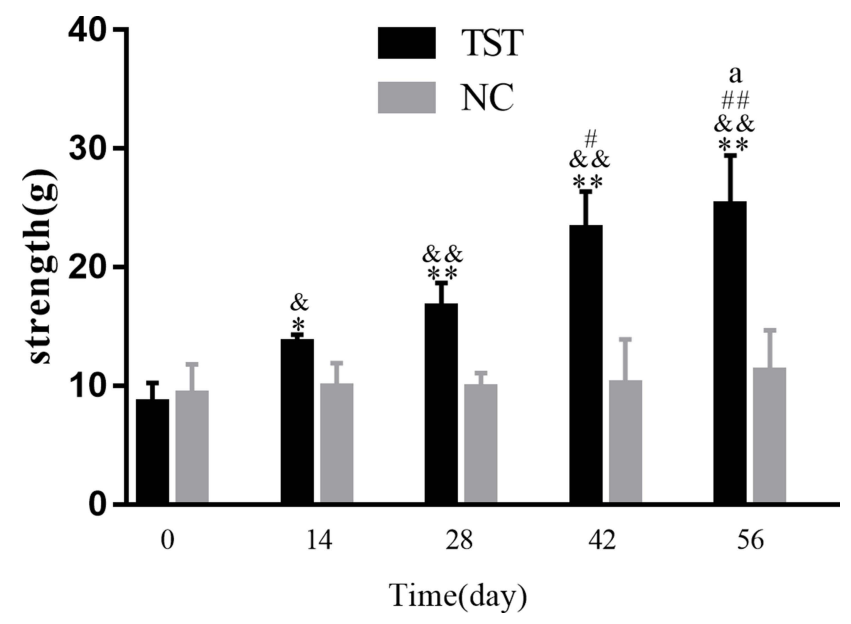

Figure 2 Comparison of MVTF between the TST group and NC group across 8-week tongue training. $* * P<0.01, * P<0.05$ compared to the NC group at the same time. ${ }^{\& \&} P<0.01,{ }^{\&} P<0.05$ compared to TST group at baseline, ${ }^{\#} P<0.01,{ }^{\#} P<0.05$ compared to TST group on day 14 , a $P<0.05$ compared to TST group on day 28 .

that in the NC group (Figure 3B). This augmentation of EMG activity persisted for $80 \mathrm{~min}$ after training on day 56 of TST (Figure 3C and $\underline{\mathrm{D}}$ ).

\section{TMS Responses of Genioglossus}

There was no statistical difference between the NC group and TST group for TMS latencies at all time points at baseline (Figure 4A). We observed that TMS latency began to decrease on day 14 , and this decline lasted for 80 min on days 28 and 56 of training (Figure 4B-D).

As the TMS amplitude varied, a significant difference between the two groups only occurred on the 28th day of training (Figure 5).

\section{Theoretical Pcrit Value of Upper Airway}

For the theoretical Pcrit value, the two groups showed similar values at baseline (Figure 6). When compared with the NC group, the TST group showed significantly decreased theoretical Pcrit value on days 28 and 56 of training, and the extent of decline on day 56 was higher than that on day 28 .

\section{Timeline of Change of TMS Latency, EMG Activity, and Theoretical Pcrit Value in TST Group}

The TST group began to present shorter TMS latency (values at 40 min after training) on day 14, higher EMG activity (values at $40 \mathrm{~min}$ after training) on day 28, and lower theoretical Pcrit value on day 56 of training when compared to baseline (Figure 7). 
A

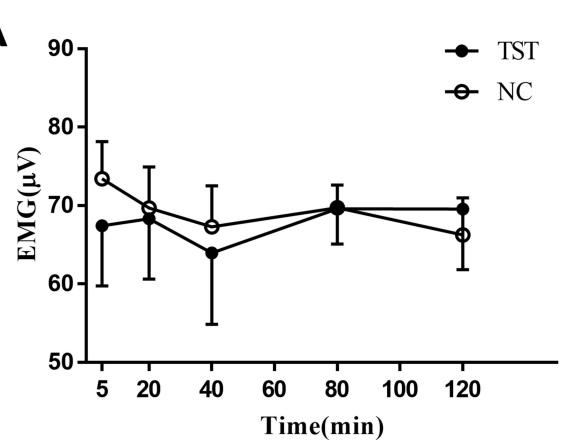

C

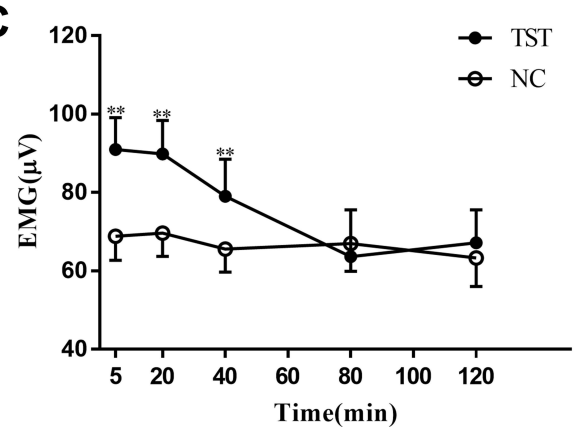

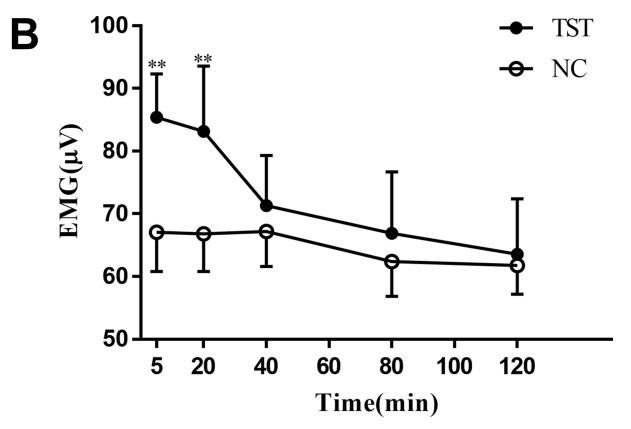

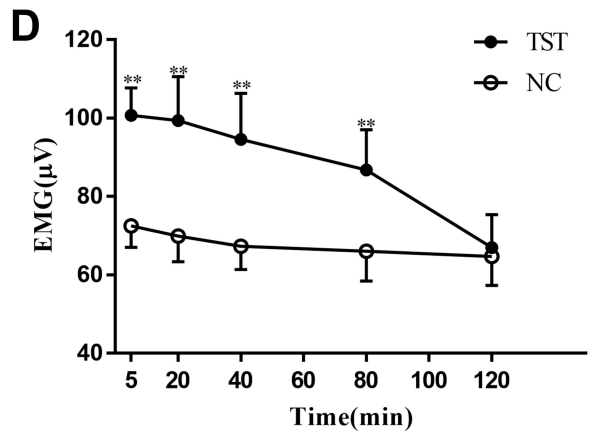

Figure 3 Comparison of genioglossus EMG activity between the TST group and NC group. (A-D) values observed on day 0, 14, 24, and 56 of training, respectively. $* *$ $P<0.01$ compared to NC group at the same time.
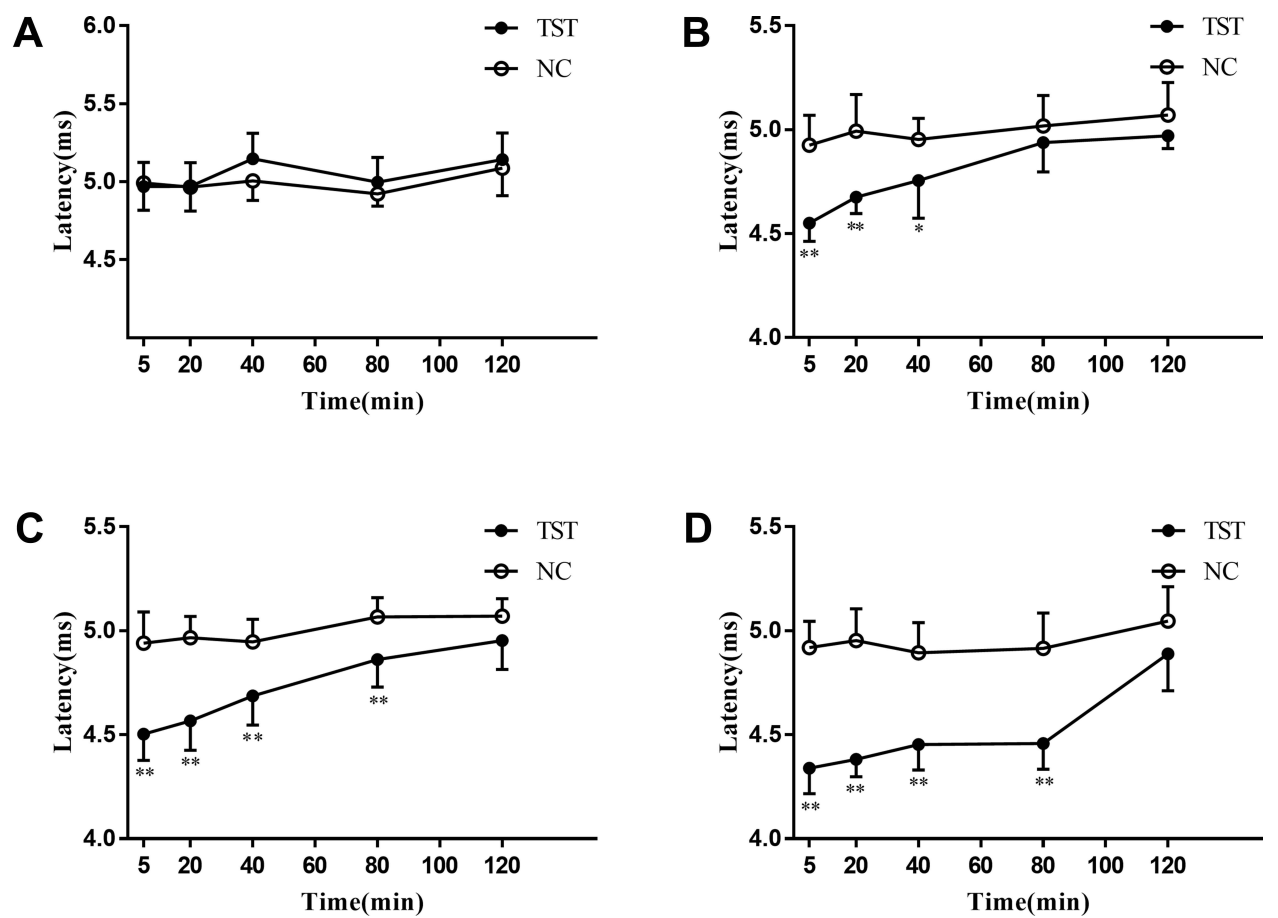

Figure 4 Comparison of TMS latencies between the TST group and NC group. (A-D) values observed on day $0,14,24$, and 56 of training, respectively. $* * P<0.01, * P<0.05$ compared to NC group at the same time.

\section{Discussion}

Indisputably, limb muscle training could increase body strength and evoke neuroplasticity. ${ }^{19}$ Then, do oropharyngeal exercises also improve upper airway collapsibility in this way? Cullins et $\mathrm{al}^{20}$ found that tongue exercise enlarged the lingual cortical motor area of rats, 

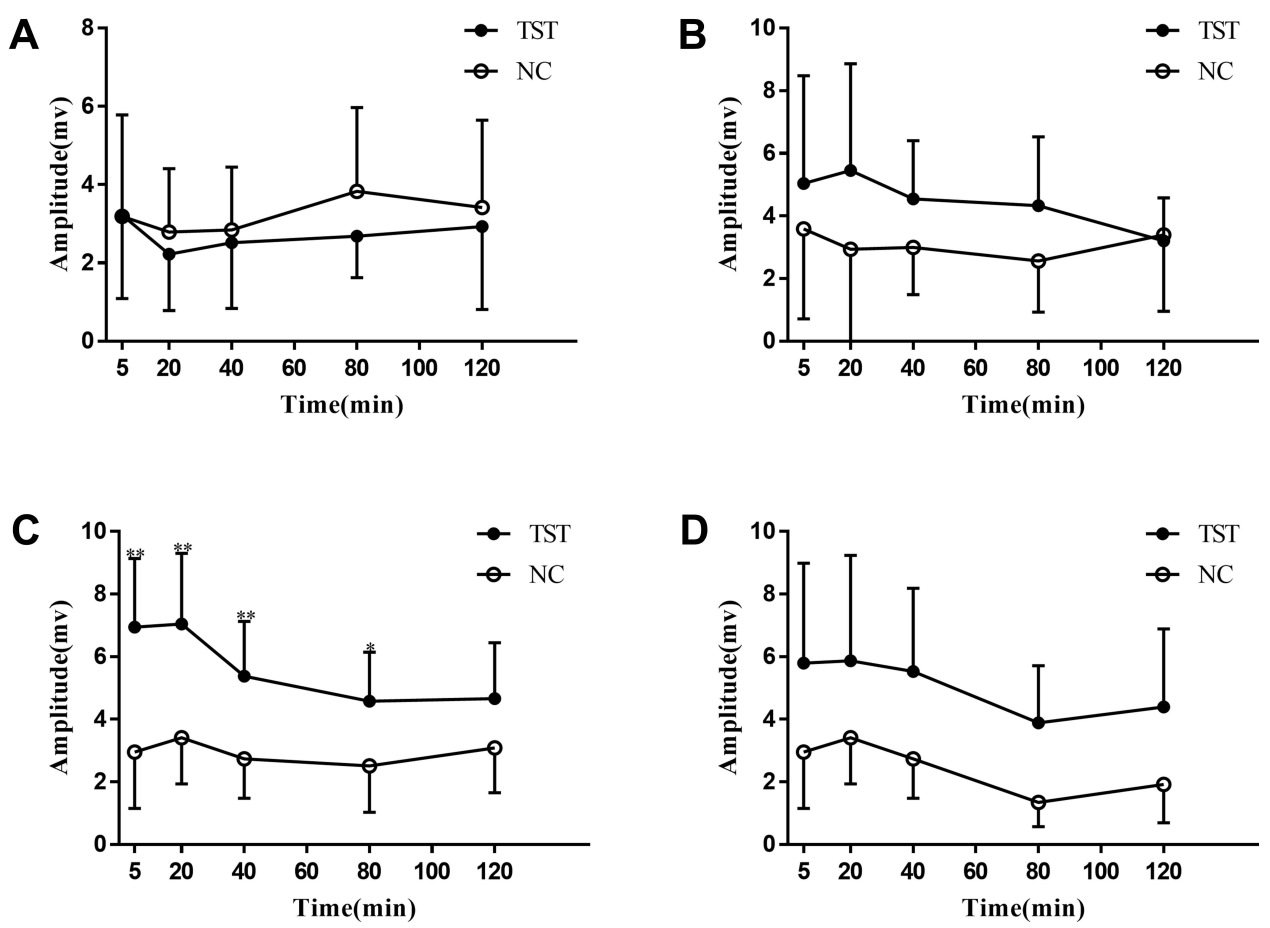

Figure 5 Comparison of TMS amplitudes between the TST group and NC group. (A-D) values observed on day $0,14,24$, and 56 of training, respectively. $* * P<0.01$, $* P<0.05$ compared to NC group at the same time.

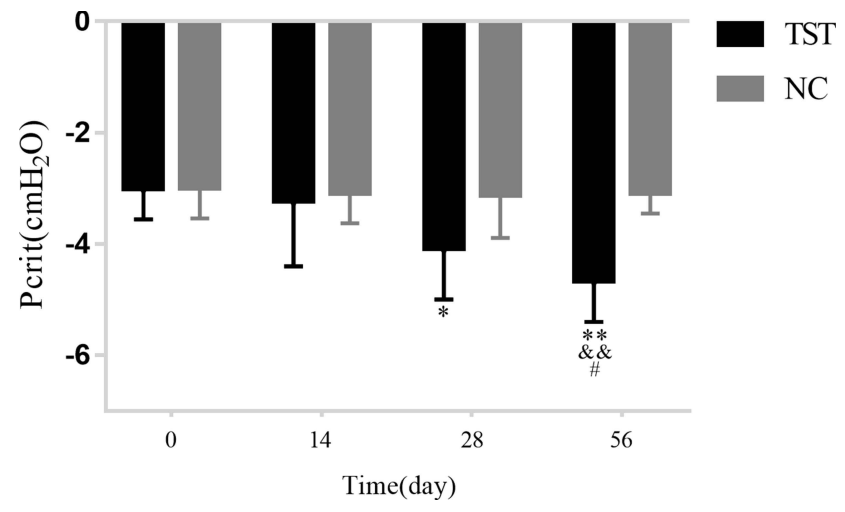

Figure 6 Comparison of theoretical Pcrit value between the TST group and NC group. $* * P<0.01, * P<0.05$ compared to NC group at the same time. ${ }^{\& \&} p<0.01$ compared to TST group at baseline. ${ }^{\#} P<0.05$ compared to TST group on day 14 of training.

indicating that tongue training could induce motor cortex plasticity. However, they only assessed it at the end of the 8 -week training. Until now, only few studies have measured the influence of progressive resistance training on the muscle function of the upper airway dilator, ${ }^{16,21}$ but no related studies explored its impact on the dynamics of the upper airway. Our study revealed that TST could not only increase tongue strength but also elevate central excitability. The elevated genioglossus corticomotor excitability and increased EMG activity persisted for $40 \mathrm{~min}$ after training on days 14 and 28 of TST, respectively. Corticomotor excitability and EMG activity increased continuously as tongue training lengthened. Furthermore, compared to before training, the TST group showed an increasing tendency toward upper airway stability on the 28th day and significantly increased on the 56th day after training. These findings revealed that an 8-week TST could improve genioglossus corticomotor excitability first, then increase genioglossus EMG activity, and eventually enhance upper airway stability. This is the first report to continuously explore the influence of TST on the central regulation of genioglossus and the collapsibility of the upper airway.

Tsai et $\mathrm{al}^{22}$ showed that 6-week treadmill running significantly enhanced the magnitude of long-term potentiation in the mouse hippocampus, and the enhancement was maintained for at least $60 \mathrm{~min}$. This facilitation effect in the central nervous system from exercise is mostly attributable to enhanced synaptic transmission efficiency, upregulated brain-derived neurotrophic factor, and promoted neurogenesis. ${ }^{23}$ On the other hand, during resistance training, neural adaptations such as increased motor unit activation, apart from improved quality of muscle fiber and increased muscle fiber size, played an important role in 


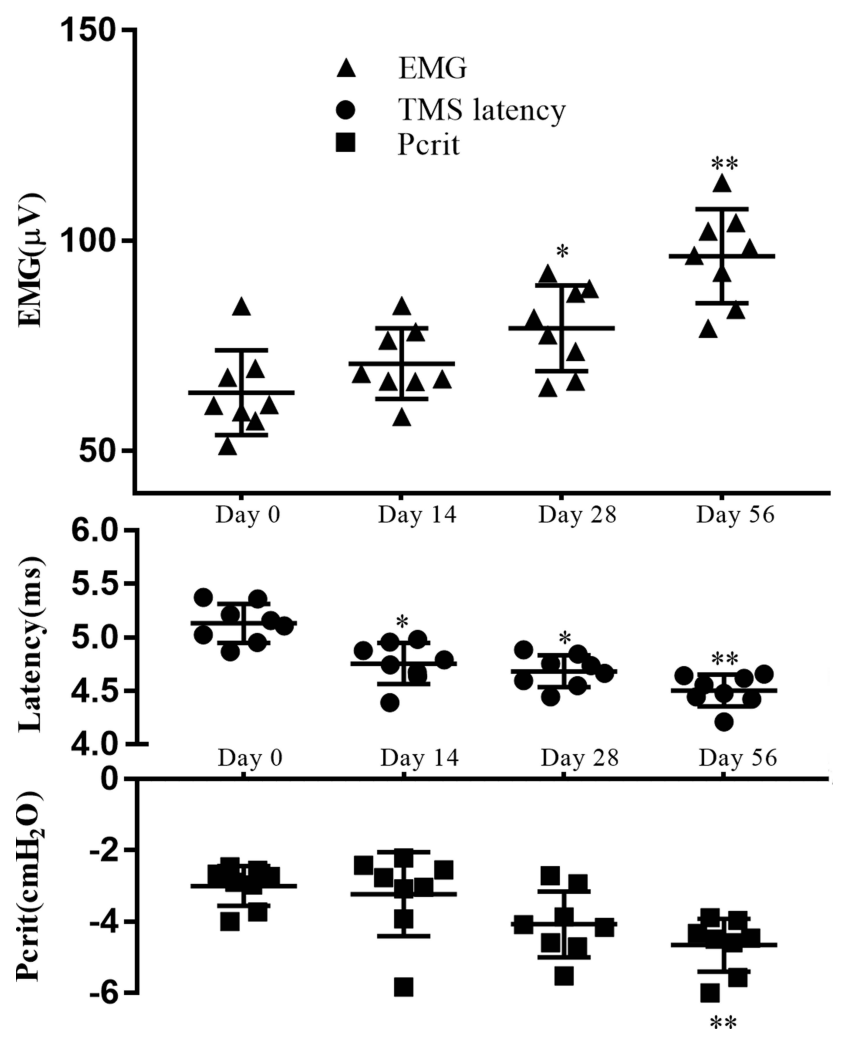

Figure 7 Comparison of variation tendency among TMS latencies (values at $40 \mathrm{~min}$ after training), EMG activity (values at 40 min after training), and theoretical Pcrit value in the TST group during training. $* * P<0.01, * P<0.05$ compared to the values detected on day 0 .

elevating muscle strength and EMG activity. ${ }^{12}$ As a form of exercise training, tongue training could also induce lingual motor cortex plasticity. ${ }^{20} \mathrm{We}$ further demonstrated that TST could induce the facilitation of the corticomotor and muscle activity of genioglossus. In addition, the intensity and duration of such excitability gradually increased with prolonged training time, which could be caused by the gradual increase in exercise intensity and difficulty over the course of training. This result is consistent with the report from Weavil et $\mathrm{al}^{24}$ that TMS amplitudes and EMG activity of thigh muscle gradually increased at greater exercise intensities during cycling.

Interestingly, the present study found that shortened TMS latency persisted longer than elevated EMG activity on days 14 and 28 of training, while both lasted $80 \mathrm{~min}$ on day 56 . Regarding the inconsistency of the extent of improvement between the TMS response and EMG activity at the early stage of training, it is likely attributable to muscle fatigue induced by resistance training. Indeed, exercise-related changes in muscle, such as depleted glycogen stores, elevated lactate accumulation, and depressed excitation-contraction coupling, could increase muscle fatigue. ${ }^{25}$ During fatiguing contractions, EMG activity gradually declines because of the dropped discharge rate of most motor units, although an augment in excitatory drive from the motor cortex to the motoneurons. ${ }^{26}$ However, as the training continues, complex neurophysiological responses and muscle architectural changes, such as increased fatigue-resistant type I muscle fibers, would compensate for fatigue. ${ }^{25}$ These alterations may account for the persistence of the improved genioglossus EMG activity, which lasted for $80 \mathrm{~min}$ on the 56th day.

Airway anatomy and neuromuscular factors, such as pattern and magnitude of activity in upper airway dilator muscles, determine upper airway collapsibility. This study found that the upper airway stability was remarkably increased after 8-week training in the training group. Our result was consistent with the outcome of Jordan et $\mathrm{al}^{27}$ that hypercapnia-induced elevation of genioglossus activity could improve airway collapsibility in OSA patients. On the other hand, after a 3-month myofunctional therapy intervention, improved modified Mallampati index was correlated with tongue and soft palate strength increases in OSA patients, indicating that an increase in muscle strength in the upper airway was beneficial for maintaining upper airway patency. ${ }^{28}$ Muscle fiber types in the genioglossus gradually shift to a type I fatigue-resistant phenotype following targeted tongue exercises. ${ }^{21}$ Thus, tongue training during the daytime seems to contribute to reducing the severity of upper airway collapse by compensating the hypotonia of the genioglossus muscle during sleep in OSA patients. ${ }^{29}$ Meanwhile, tongue training may improve the function of other upper airway dilator muscles (such as the geniohyoid, hyoglossus, and stylohyoid) and further improve upper airway stability by enhancing synergy between them and genioglossus. ${ }^{29}$ These findings revealed that tongue training could be an effective treatment for OSA by improving upper airway collapsibility. On the other hand, the low workload and occurrence of muscle fatigue might illustrate the absence of improved upper airway stability at the early stage of training. However, as the training continued, upper airway stability eventually significantly enhanced late in training due to improved muscle fatigue resistance and gradually elevated corticomotor excitability. These results indicate that patients with OSA might benefit from TST. Although oropharyngeal training is thought to improve upper airway function, its effectiveness in the treatment of OSA and the suitable target groups remain uncertain. The present study confirmed that TST could improve upper airway stability by enhancing the central regulation of genioglossus. 
From a methodological perspective, we individually customized the force threshold for each rat every training day, and first introduced muscle total work to quantify the daily total training volume based on previous studies. These settings could ensure exercise-related benefits to a greater extent. Meanwhile, we first explored upper airway collapsibility noninvasively and dynamically in a TST rat model. However, this study has several limitations. First, given the effect of exercise training on tongue muscle histomorphology, ${ }^{16}$ it is possible that there are interactions between muscle properties, such as muscle hypertrophy and fatigue indices, and central regulation of muscle after training that we did not assess in the present study. Future experiments are needed to distinguish such interactions and their effect on the stability of the upper airway. Second, the TMS response is a complex integration of corticospinal excitability and muscle activation, and many factors, including muscle statuses, can influence the response. However, many previous studies evaluated central excitability using TMS. The present study also observed significantly shortened TMS latencies of rats in the TST group after tongue training. In the future, it is necessary to precisely focus on genioglossus motor cortical changes following tongue training, including its structural alterations. Third, with reference to the method of previous studies, ${ }^{30}$ we calculated the theoretical Pcrit value by measuring the upper airway pressure and instantaneous airflow across the oropharynx. Unfortunately, we did not validate the theoretical Pcrit value with conventional methodology. However, we did verify the collapse site of the upper airway by 3-dimensional computed tomography. ${ }^{14}$ Our results indicated that this noninvasive method of measuring the Pcrit had good stability and repeatability. The present study did find the difference of theoretical Pcrit value in rats before and after training. Fourth, the rats in the present study only participated in an 8-week TST program. As the duration of exercise training in most clinical trials is 3 months, it is necessary to assess the effect of long-term training. In addition, to better guide individual treatment in patients with OSA, studies are needed to explore the morphological characteristics and biological markers of responders to TST intervention.

In conclusion, this study confirmed that TST could improve corticomotor excitability of genioglossus and elevate genioglossus EMG activity, with the extent and duration of this increase growing as tongue training is lengthened. Ultimately, TST enhanced the upper airway stability of rats after 8-week training. OSA is a chronic disease and the duration of exercise training in most clinical trials is 3 months or more, and taking this into consideration, TST might provide a new method to improve the AHI of OSA patients. Future studies are needed to identify the appropriate target patients and explore individualized training doses.

\section{Acknowledgments}

We would like to thank Editage for English language editing.

\section{Author Contributions}

All authors contributed to data analysis, drafting or revising the article, have agreed on the journal to which the article will be submitted, gave final approval of the version to be published, and agree to be accountable for all aspects of the work.

\section{Funding}

This study was funded by the National Natural Science Foundation of China (81670085).

\section{Disclosure}

The authors report no conflicts of interest in this work. The abstract of this manuscript has been presented at the 3rd Sleep Congress of the Asian Society of Sleep Medicine on May 14, 2021.

\section{References}

1. Mezzanotte W, Tangel D, White D. Influence of sleep onset on upper-airway muscle activity in apnea patients versus normal controls. Am J Respir Crit Care Med. 1996;153(6):1880-1887. doi:10.1164/ajrccm.153.6.8665050

2. Eckert DJ, White DP, Jordan AS, Malhotra A, Wellman A. Defining phenotypic causes of obstructive sleep apnea. Identification of novel therapeutic targets. Am J Respir Crit Care Med. 2013;188 (8):996-1004. doi:10.1164/rccm.201303-0448OC

3. Fogel R, Trinder J, White D, et al. The effect of sleep onset on upper airway muscle activity in patients with sleep apnoea versus controls. J Physiol. 2005;564:549-562. doi:10.1113/jphysiol.2005.083659

4. Wang W, Kang J, Kong D. The central motor conductivity of genioglossus in obstructive sleep apnoea. Respirology. 2010;15 (8):1209-1214. doi:10.1111/j.1440-1843.2010.01858.x

5. Series F, Wang W, Similowski T. Corticomotor control of the genioglossus in awake OSAS patients: a transcranial magnetic stimulation study. Respir Res. 2009;10:74. doi:10.1186/1465-9921-10-74

6. Malhotra A, Pillar G, Fogel R, Beauregard J, Edwards J, White D. Upper-airway collapsibility: measurements and sleep effects. Chest. 2001;120(1):156-161. doi:10.1378/chest.120.1.156

7. Rueda J, Mugueta-Aguinaga I, Vilaró J, Rueda-Etxebarria M. Myofunctional therapy (oropharyngeal exercises) for obstructive sleep apnoea. Cochrane Database Syst Rev. 2020;11:CD013449.

8. Verma R, Johnson JJ, Goyal M, Banumathy N, Goswami U, Panda N. Oropharyngeal exercises in the treatment of obstructive sleep apnoea: our experience. Sleep Breathing. 2016;20(4):1193-1201. doi:10.1007/s11325-016-1332-1

9. Robbins J, Gangnon R, Theis S, Kays S, Hewitt A, Hind J. The effects of lingual exercise on swallowing in older adults. J Am Geriatr Soc. 2005;53 (9):1483-1489. doi:10.1111/j.1532-5415.2005.53467.x

10. Yeates E, Molfenter S, Steele C. Improvements in tongue strength and pressure-generation precision following a tongue-pressure training protocol in older individuals with dysphagia: three case reports. Clin Interv Aging. 2008;3(4):735-747. doi:10.2147/CIA.S3825 
11. Rousseau E, Melo-Silva CA, Gakwaya S, Sériès F. Effects of one-week tongue-task training on sleep apnea severity: a pilot study. Can Respir J. 2015;22:176-178.

12. Gabriel D, Kamen G, Frost G. Neural adaptations to resistive exercise: mechanisms and recommendations for training practices. Sports Med. 2006;36(2):133-149. doi:10.2165/00007256-200636020-00004

13. Sforza E, Petiau C, Weiss T, Thibault A, Krieger J. Pharyngeal critical pressure in patients with obstructive sleep apnea syndrome. Clinical implications. Am J Respir Crit Care Med. 1999;159 (1):149-157. doi:10.1164/ajrccm.159.1.9804140

14. Meng Y, Li W, Zou Y, et al. How does chronic intermittent hypoxia influence upper airway stability in rats? Nat Sci Sleep. 2020;12:749-758. doi:10.2147/NSS.S249948

15. Krekeler BN, Weycker JM, Connor NP. Effects of tongue exercise frequency on tongue muscle biology and swallowing physiology in a rat model. Dysphagia. 2020;35:1-17.

16. Connor NP, Russell JA, Wang H, Jackson MA, Mann L, Kluender K. Effect of tongue exercise on protrusive force and muscle fiber area in aging rats. $J$ Speech Lang Hearing Res. 2009;52(3):732-744. doi:10.1044/1092-4388(2008/08-0105)

17. Medicine A. American College of Sports Medicine position stand. Progression models in resistance training for healthy adults. Med Sci Sports Exerc. 2009;41(3):687.

18. Nie X, Zhou L, Wang A, et al. Noradrenergic activation of hypoglossal nucleus modulates the central regulation of genioglossus in chronic intermittent hypoxic rats. Front Neurol. 2017;8:171. doi:10.3389/fneur.2017.00171

19. Vilela T, Muller A, Damiani A, et al. Strength and aerobic exercises improve spatial memory in aging rats through stimulating distinct neuroplasticity mechanisms. Mol Neurobiol. 2017;54(10):7928-7937. doi:10.1007/s12035-016-0272-x

20. Cullins MJ, Wenninger JM, Cullen JS, Russell JA, Kleim JA, Connor NP. Tongue force training induces plasticity of the lingual motor cortex in young adult and aged rats. Front Neurosci. 2019;13:1355. doi:10.3389/fnins.2019.01355
21. Kletzien H, Russell JA, Leverson GE, Connor NP. Differential effects of targeted tongue exercise and treadmill running on aging tongue muscle structure and contractile properties. J Appl Physiol. 2013;114 (4):472-481. doi:10.1152/japplphysiol.01370.2012

22. Tsai $\mathrm{SF}, \mathrm{Ku} \mathrm{NW}$, Wang $\mathrm{TF}$, et al. Long-term moderate exercise rescues age-related decline in hippocampal neuronal complexity and memory. Gerontology. 2018;64(6):551-561. doi:10.1159/000488589

23. Ma Q. Beneficial effects of moderate voluntary physical exercise and its biological mechanisms on brain health. Neurosci Bull. 2008;24 (4):265-270. doi:10.1007/s12264-008-0402-1

24. Weavil JC, Sidhu SK, Mangum TS, Richardson RS, Amann M. Intensity-dependent alterations in the excitability of cortical and spinal projections to the knee extensors during isometric and locomotor exercise. Am J Physiol Regu Int Comparative Physiol. 2015;308(12):R998-R1007. doi:10.1152/ajpregu.00021.2015

25. Ament W, Verkerke G. Exercise and fatigue. Sports Med. 2009;39 (5):389-422. doi:10.2165/00007256-200939050-00005

26. Garland S, Enoka R, Serrano L, Robinson G. Behavior of motor units in human biceps brachii during a submaximal fatiguing contraction. $J \mathrm{Appl}$ Physiol. 1994;76(6):2411-2419. doi:10.1152/jappl.1994.76.6.2411

27. Jordan AS, White DP, Owens RL, et al. The effect of increased genioglossus activity and end-expiratory lung volume on pharyngeal collapse. J Appl Physiol. 2010;109(2):469-475. doi:10.1152/ japplphysiol.00373.2010

28. Diaféria G, Santos-Silva R, Truksinas E, et al. Myofunctional therapy improves adherence to continuous positive airway pressure treatment. Sleep Breath. 2017;21(2):387-395. doi:10.1007/s11325-016-1429-6

29. de Felício CM, da Silva Dias FV, Trawitzki LVV. Obstructive sleep apnea: focus on myofunctional therapy. Nat Sci Sleep. 2018;10:271. doi:10.2147/NSS.S141132

30. Nakano H, Magalang U, Lee S, Krasney J, Farkas G. Serotonergic modulation of ventilation and upper airway stability in obese Zucker rats. Am J Respir Crit Care Med. 2001;163(5):1191-1197. doi:10.1164/ajrccm.163.5.2004230

\section{Publish your work in this journal}

Nature and Science of Sleep is an international, peer-reviewed, open access journal covering all aspects of sleep science and sleep medicine, including the neurophysiology and functions of sleep, the genetics of sleep, sleep and society, biological rhythms, dreaming, sleep disorders and therapy, and strategies to optimize healthy sleep.
The manuscript management system is completely online and includes a very quick and fair peer-review system, which is all easy to use. Visit http://www.dovepress.com/testimonials.php to read real quotes from published authors. 